OPEN ACCESS

Edited by:

Min Wu,

Sichuan University, China

Reviewed by:

Xianwen Wang,

Anhui Medical University, China

Li-Min Zhu,

Donghua University, China

Hailin Cong,

Qingdao University, China

*Correspondence:

Jian Dong

dongjian@sdfmu.edu.cn

Haijun Wang

hiwang@sdfmu.edu.ch

Xiao Sun

sunxiao@sdfmu.edu.cn

${ }^{\dagger}$ These authors have contributed equally to this work

Specialty section:

This article was submitted to

Cancer Imaging and Image-directed Interventions,

a section of the journal

Frontiers in Oncology

Received: 23 January 2022 Accepted: 08 February 2022

Published: 03 March 2022

Citation:

Zou X, Ma G, Zhu P, Cao Y,

Sun $X$, Wang $H$ and Dong $J$ (2022) A Polydopamine-Coated

Platinum Nanoplatform for Tumor-

Targeted Photothermal Ablation

and Migration Inhibition.

Front. Oncol. 12:860718.

doi: 10.3389/fonc.2022.860718

\section{A Polydopamine-Coated Platinum Nanoplatform for Tumor-Targeted Photothermal Ablation and Migration Inhibition}

\author{
Xianwen Zou ${ }^{1 \dagger}$, Guiqi Ma ${ }^{1+}$, Pengyu Zhu ${ }^{1}$, Yutao Cao ${ }^{1}$, Xiao Sun ${ }^{1 *}$, Haijun Wang ${ }^{2 *}$ \\ and Jian Dong ${ }^{1 *}$ \\ 1 Institute of Optical Functional Materials for Biomedical Imaging, School of Chemistry and Pharmaceutical Engineering, \\ Shandong First Medical University and Shandong Academy of Medical Sciences, Taian, China, 2 School of Life Sciences, \\ Shandong First Medical University and Shandong Academy of Medical Sciences, Taian, China
}

In this work, Arg-Gly-Asp (RGD) peptide-coupled polydopamine-modified mesoporous platinum nanoparticles (mPt@PDA-RGD NPs) were developed for targeted photothermal therapy (PTT) and migration inhibition of SKOV-3 cells. mPt@PDA-RGD NPs with obvious core/shell structure demonstrated high photothermal performance under 808-nm nearinfrared (NIR) laser irradiation. mPt@PDA-RGD NPs with favorable biocompatibility exhibited remarkable SKOV-3 inhibition ability under NIR laser irradiation. Moreover, compared to mPt@PDA NPs, the RGD-functionalized NPs achieved more tumor uptake and PTT performance, which was attributed to the specific interaction between RGD of NPs and $\alpha_{v} \beta_{3}$ integrin overexpressed by SKOV-3. Importantly, cell scratch experiments indicated that the photothermal effect of mPt@PDA-RGD NPs can effectively inhibit the migration of surviving SKOV-3 cells, which was assigned to disturbance of the actin cytoskeleton of SKOV-3. Thus, mPt@PDA-RGD NPs presented great potential for targeted tumor photothermal ablation and migration inhibition.

Keywords: platinum nanoplatform, RGD target, PTT, photothermal ablation, migration inhibition

\section{INTRODUCTION}

Tumor has emerged as a serious threat to human health. As a popular noninvasive treatment for local tumors, photothermal therapy (PTT) has attracted great attention worldwide (1-4). PTT usually depends on the photosensitizer to create enough heat to inhibit tumor or even kill them and has been recognized as an effective and minimally invasive treatment strategy for primary tumors $(5,6)$. Compared to traditional treatments of cancer, such as radiotherapy and chemotherapy, PTT has certain advantages including less invasiveness, fewer side effects, and higher specificity $(7,8)$. The photosensitizer is expected to be nontoxic and relatively safe to cells in the absence of any laser irradiation. In addition, due to the precise control of laser irradiation 
parameters (such as position, laser wavelength, irradiation time, and light intensity), PTT can show great selectivity and minimal side effects $(9,10)$.

The development of nanomedicine improves the diagnosis and treatment efficiency of tumors (11-14). Nanomaterials with plasmonic nanostructure can be well activated by near-infrared (NIR) for PTT of cancer $(15,16)$. For example, gold nanoparticles (GNPs) have attracted much attention due to their high photothermal conversion efficiency $(17,18)$. Gold nanostructures, including gold nanorods (19), gold nanoshells (20), gold nanocages (21), and hollow gold nanospheres (22), have been most extensively studied as PTT reagents. Currently, the application of platinum $(\mathrm{Pt})$ nanomaterials has attracted more and more attention in the biomedical field. Pt nanomaterials have extensive light absorption in the NIR region. For example, Manikandan et al. (23) reported for the first time that polyvinylpyrrolidone (PVP)-coated Pt nanoparticles (NPs) have photothermal conversion capability. However, this inorganic nanoplatform often faces problems of low biocompatibility when used in the biomedical field.

As a promising material for the preparation of multifunctional nanomaterials for cancer treatment, polydopamine (PDA) has attracted more attention in the biomedical field (24-26). Dopamine is a kind of biomimetic mussel adhesive protein, which can self-polymerize and spontaneously deposit onto various materials under alkaline $\mathrm{pH}$ conditions, forming a controllable PDA layer $(27,28)$. PDA coating has special adhesion ability and good biocompatibility (29). In addition, PDA, with a lot of catechol and amino groups on its outer surface, can contribute to nanomaterials' functionalization of cellspecific ligands and biocompatibility (30). RGD peptide is related to tumor progression and less expressed in mature vascular endothelial cells and normal organ systems. Thus, RGD peptide has been used to modify nanomaterials to improve receptor-mediated cell adhesion by recognizing integrin $\alpha_{v} \beta_{3}$ of cancer cells, thereby promoting endocytosis (31-33).

In this work, Arg-Gly-Asp (RGD) peptide-coupled PDAmodified mesoporous Pt (mPt) (mPt@PDA-RGD NPs), with mPt as core, the self-polymerizing dopamine as the shell, and the grafted RGD as targeting ligands, were prepared (Scheme 1). Under 808nm laser irradiation, mPt@PDA-RGD NPs exhibited excellent biocompatibility, chemotaxis of tumors, and efficient SKOV-3 inhibition ability. Immunofluorescence imaging showed that the actin cytoskeleton of SKOV-3 was destroyed and the migration of cancer cells was effectively inhibited after PTT. Thus, mPt@PDARGD NPs present great potential for targeted photothermal cancer treatments.

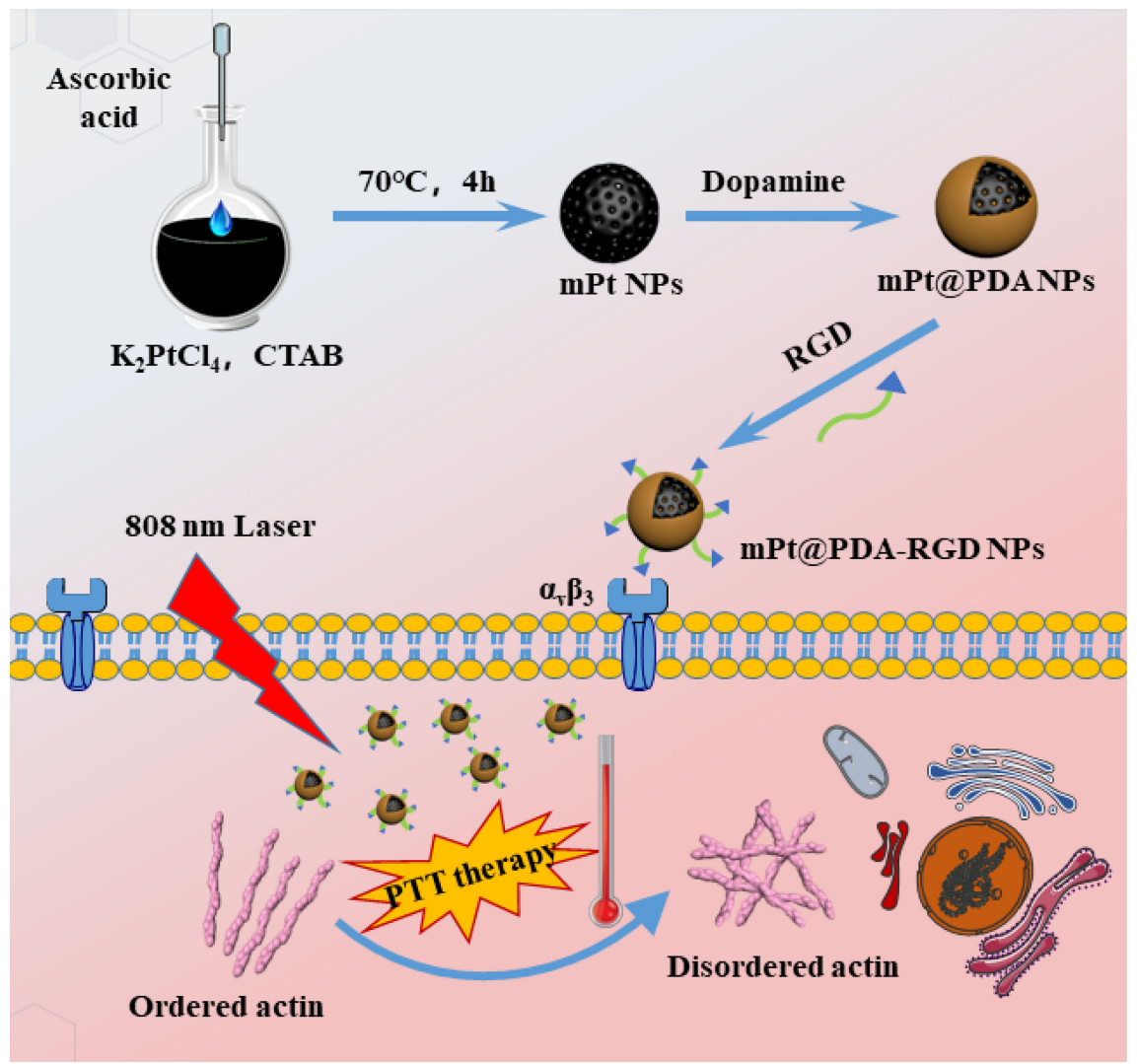

SCHEME 1 | The synthesis of Arg-Gly-Asp peptide-coupled polydopamine-modified mesoporous platinum nanoparticles (mPt@PDA-RGD NPs) and their application in targeted cancer therapy. 


\section{MATERIALS AND METHODS}

\section{Materials}

Potassium tetrachloroplatinate (II) $\left(\mathrm{K}_{2} \mathrm{PtCl}_{4}\right)$, hexadecyltrimethylammonium bromide (CTAB), ascorbic acid, dopamine hydrochloride (DA.HCl), and RGD were obtained from Aladdin. Tris-HCl buffer (10 mM, pH 8.5) was gained from Phygene (China). The cell counting kit-8 (CCK-8) was bought from Beyotime Biotechnology. Calcein AM and PI were obtained from KeyGen Biotech Co. (China). Cancer cell line SKOV-3 was obtained from the Type Culture Collection of the Chinese Academy of Sciences. Dulbecco's modified Eagle's medium (DMEM) was obtained from Procell (Wuhan, China). Penicillin-streptomycin, Fetal bovine serum (FBS), Trypsin-Ethylene Diamine Tetraacetic Acid (EDTA), and Phosphate buffered solution (PBS) were obtained from Solarbio Science \& Technology Co. (China). All chemicals were purchased from commercial suppliers and directly used.

\section{Fabrication of mPt@PDA-RGD Nanocomposite}

Pt NPs with porous structure were prepared via a simple chemical reduction process (34). Briefly, $\mathrm{K}_{2} \mathrm{PtCl}_{4}$ solution $(10 \mathrm{mM}, 36 \mathrm{ml})$ and CTAB solution $(100 \mathrm{mM}, 90 \mathrm{ml})$ were mixed together at $70^{\circ} \mathrm{C}$ for $0.5 \mathrm{~h}$. Then, ascorbic acid $(20 \mathrm{mM}, 54 \mathrm{ml})$ was added to the mixture, and the solution was further heated at $70^{\circ} \mathrm{C}$ for $3 \mathrm{~h}$. Excess $\mathrm{CTAB}$ was washed away with absolute ethanol; next, the nanoparticles were dispersed in deionized water. mPt@PDA NPs were synthesized by reaction of $\mathrm{mPt} N \mathrm{NPs}$ and dopamine in a 5:1 ratio in tris- $\mathrm{HCl}$ buffer $(10 \mathrm{mM}, \mathrm{pH} 8.5)$ for $1 \mathrm{~h}$. For the synthesis of the mPt@PDA-RGD, tris buffer (5 ml) containing RGD (10 mg) was mixed with mPt@PDA NPs (20 mg in $35 \mathrm{ml}$ of tris- $\mathrm{HCl}$ buffer) for $30 \mathrm{~min}$ of ultrasonic treatment, followed by stirring for $12 \mathrm{~h}$.

\section{Characterization}

A high-resolution transmission electron microscopy (HRTEM; TecnaiG2 F20, FEI, Ohio, USA) was used to observe the morphology of mPt NPs and mPt@PDA NPs. The surface area and pore size of the $\mathrm{mPt}$ NPs were measured by using an automated gas sorption analyzer (Quantachrome, Autosorb-iQ). X-ray diffraction (XRD) pattern was obtained on a powder X-Ray diffractometer (Bruker, D8 ADVANCE). X-ray photoelectron spectroscopy (XPS) was performed using a multifunctional imaging electron spectrometer (Thermo ESCALAB 250XI). UV-Vis absorption spectra were taken on a U-3900 spectrophotometer (Hitachi, Japan). The hydrodynamic sizes and Z-potential were recorded by Malvern Zetasizer NanoZS90 (Malvern, United Kingdom). Fourier transform infrared spectroscopy (FTIR) spectra were tested on IRAffinity-1S (Shimadzu, Japan). Fluorescence imaging photographs were obtained by confocal laser scanning microscope (FV-3000, Olympus). The uptake of Pt element in SKOV-3 cells was measured by Inductively Coupled Plasma-Optical Emission Spectrometer (ICP-OES).

\section{Photothermal Properties of mPt@PDA- RGD Nanocomposite}

To test the photothermal properties of nanomaterials, $0.2 \mathrm{ml}$ of mPt@ PDA-RGD NP solution with various concentrations $(0,25,50,100$, $150,200 \mu \mathrm{g} / \mathrm{ml}$ ) was treated with $808-\mathrm{nm}$ laser at power densities of 1.5 $\mathrm{W} / \mathrm{cm}^{2}$ for $5 \mathrm{~min}$. In addition, mPt@PDA-RGD NP solution (0.2 ml) of equal concentration $(100 \mu \mathrm{g} / \mathrm{ml})$ was treated with 808 -nm lasers $(0.5$, 1,1.5, and $2 \mathrm{~W} / \mathrm{cm}^{2}$ ). To study the thermal stability, $0.2 \mathrm{ml}$ of mPt@ PDA-RGD NP solution $(100 \mu \mathrm{g} / \mathrm{ml})$ was irradiated at $1.5 \mathrm{~W} / \mathrm{cm}^{2}$ for four ON/OFF cycles. The corresponding temperatures are recorded per second with an infrared thermometer (CEM, DT-8891E).

\section{Cytotoxicity Test}

To evaluate the toxicity of mPt NPs and mPt@PDA-RGD NPs by CCK-8 assay, first, SKOV-3 cells were seeded into a 96-well plate $\left(1 \times 10^{4}\right.$ cells per well $)$ overnight. Then, the cells were treated with mPt NPs or mPt@PDA-RGD NPs at 0, 10, 20, 40, 80, and $100 \mu \mathrm{g} /$ $\mathrm{ml}$ in the cell incubator for $24 \mathrm{~h}$. After washing with PBS, CCK-8 solution $(10 \mu \mathrm{l})$ accompanied by culture medium $(100 \mu \mathrm{l})$ was added to each well. After 2-h incubation without light, the absorbance was measured at $450 \mathrm{~nm}$. Each experiment had 3 parallel groups.

\section{In Vitro Cellular Uptake Measurement}

To investigate the cellular uptake of mPt@PDA-RGD NPs, SKOV-3 cells were transferred to a 6 -well plate $\left(1 \times 10^{5}\right.$ cells per well). After 24-h incubation, the old medium was replaced with fresh medium accompanied by mPt@PDA NPs or mPt@PDA-RGD NPs; both of their concentrations were $25 \mu \mathrm{g} / \mathrm{ml}$. After 2-h or 4-h incubation, the cells were washed with PBS, treated with trypsin, and digested with $5 \mathrm{ml}$ of aqua regia. Finally, Pt content in each sample was tested by ICP-OES. Each experiment was performed three times.

\section{Photothermal Therapy of Cancer Cells}

In simple terms, SKOV-3 cells were grown on 96 -well plates $(5 \times$ $10^{3}$ cells per well). There were four groups of the phototoxicity tests (control, laser, mPt@PDA-RGD NPs, and mPt@PDA-RGD NPs +laser). The control group was left untreated. The cells in the laser group were irradiated under $808-\mathrm{nm}$ NIR $\left(1.5 \mathrm{~W} / \mathrm{cm}^{2}\right)$ for $5 \mathrm{~min}$. The remaining two groups received mPt@PDA-RGD NPs $(50 \mu \mathrm{g} /$ $\mathrm{ml}$ ) and incubated for $24 \mathrm{~h}$. Next, the cells were respectively irradiated without or with $808-\mathrm{nm}$ laser $\left(1.5 \mathrm{~W} / \mathrm{cm}^{2}\right)$ for $5 \mathrm{~min}$. Finally, the viabilities of SKOV-3 cells were examined by CCK-8 kit.

\section{Cytoskeleton Staining}

SKOV-3 cells were treated with mPt@PDA-RGD NPs (0, 50, $100 \mu \mathrm{g} /$ $\mathrm{ml})$ and irradiated with $808 \mathrm{~nm}\left(1.5 \mathrm{~W} / \mathrm{cm}^{2}\right)$ for $50 \mathrm{~s}$, then fixed with $4 \%$ paraformaldehyde $15 \mathrm{~min}$, permeabilized with Triton X-100 (0.2\%), and blocked with 5\% Bovine Serum Albumin (BSA) at room temperature for $1 \mathrm{~h}$. Then washed with PBS and incubated with TRITC Phalloidin (MKbio, Shanghai, China) for $1 \mathrm{~h}$ and further stained by Hoechst $33342(10 \mu \mathrm{l} ; 10 \mu \mathrm{g} / \mathrm{ml})$. The labeled slides were mounted with Prolong ${ }^{\mathrm{TM}}$ Gold Antifade Reagent with 4',6Diamidino-2-phenylindole Dihydrochloride (DAPI) (Invitrogen, Carlsbad, USA). The cells were imaged by using confocal laser scanning microscope (CLSM; FV-3000, Olympus, Japan).

\section{Western Blot}

SKOV-3 cells were incubated with mPt@PDA-RGD NPs $(0,50$, $100,200 \mu \mathrm{g} / \mathrm{ml})$ for 6 hours, then irradiated with $808 \mathrm{~nm}(1.5 \mathrm{~W} /$ $\mathrm{cm}^{2}$ ) for $50 \mathrm{~s}$. After washing with cold PBS, cell lysate (Servicebio, China) and protease inhibitor were used to lyse the cancer cells, followed by incubation for $30 \mathrm{~min}$ at $4^{\circ} \mathrm{C}$. Then, proteins were extracted by centrifuging. The extracted proteins were separated by 
$10 \%$ sodium dodecyl sulfate - polyacrylamide gel electrophoresis (SDS-PAGE) (Epizyme, China). Then, the proteins were transferred to polyvinylidene fluoride (PVDF). The membranes were incubated with primary antibodies (actin 1:2,000, glyceraldehyde-3-phosphate dehydrogenase (GAPDH) 1:1,000) at $4^{\circ} \mathrm{C}$ for $12 \mathrm{~h}$. After washing three times, the membranes were incubated with secondary antibodies for $1 \mathrm{~h}$, then washed with PBST, and the membranes were treated with chemiluminescence agents.

\section{Cell Migration Assay}

SKOV-3 cells were inoculated in culture inserts to create a clean cell gap, and mPt@PDA-RGD NPs at a concentration of $50 \mu \mathrm{g} / \mathrm{ml}$ or $100 \mu \mathrm{g} / \mathrm{ml}$ were added to the holes when the cells have grown to $90 \%$ to form a single layer and incubated for $6 \mathrm{~h}$, then cleaned with PBS, and treated with $808 \mathrm{~nm}\left(1.5 \mathrm{~W} / \mathrm{cm}^{2}\right)$ for $50 \mathrm{~s}$. Cell migration was monitored $24 \mathrm{~h}$ after culture.

\section{Annexin V/ Propidium Assay}

Annexin V/PI kit was utilized to determine apoptosis and necrosis rate. SKOV-3 cells were grown in 24-well plates and incubated for 12 h. The mPt@PDA-RGD NPs were added and incubated with the cells for $24 \mathrm{~h}$. Then, the medium was refreshed and exposed to 808$\mathrm{nm}$ laser $\left(1.5 \mathrm{~W} / \mathrm{cm}^{2}\right)$ for $5 \mathrm{~min}$. The cells were digested with trypsin and dispersed with binding buffer. After Annexin V and PI staining, the fluorescence intensity of cells was observed by flow cytometer (BD Accuri ${ }^{\mathrm{TM}}$ C6 Plus).

\section{In Vitro Photothermal Induced Cell Death}

SKOV-3 cells were grown in glass bottom dishes. The mPt@PDA NPs or mPt@PDA-RGD NPs $(100 \mu \mathrm{g} / \mathrm{ml})$ were added, and cells were incubated for $4 \mathrm{~h}$. Next, the cells were washed with PBS and refreshed with new medium and treated with 808-nm laser (1.5 W/ $\mathrm{cm}^{2}$ ) for $5 \mathrm{~min}$. Medium was removed, and $200 \mu \mathrm{l}$ of Calcein AM/ PI working solution was added for $30 \mathrm{~min}$ to stain cells. After careful cleaning with PBS, living and dead cells were analyzed by CLSM.

\section{Statistical Analysis}

All results are presented as mean \pm standard deviation (SD), and between-group comparisons were evaluated using one-way ANOVA. A $\mathrm{p}$ value $<0.05$ indicates statistical significance, and data are represented as ${ }^{*}$ for $\mathrm{p}<0.05,{ }^{* *}$ for $\mathrm{p}<0.01,{ }^{* * *}$ for $\mathrm{p}<0.001$, and ${ }^{\star * * \star}$ for $\mathrm{p}<0.0001$. NS stands for not statistically significant.

\section{RESULTS AND DISCUSSION}

\section{Preparation and Characterization of mPt@ PDA-RGD Nanoparticles}

TEM image (Figure 1A) shows that the average diameter of the synthesized mPt NPs was about $70 \mathrm{~nm}$, and the morphology of the synthesized $\mathrm{mPt}$ NPs was obviously porous (Figure 1B). The

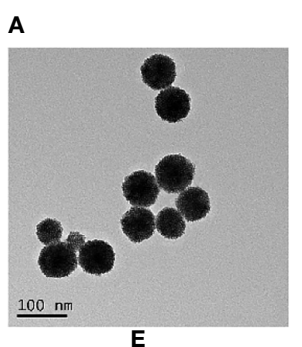

B
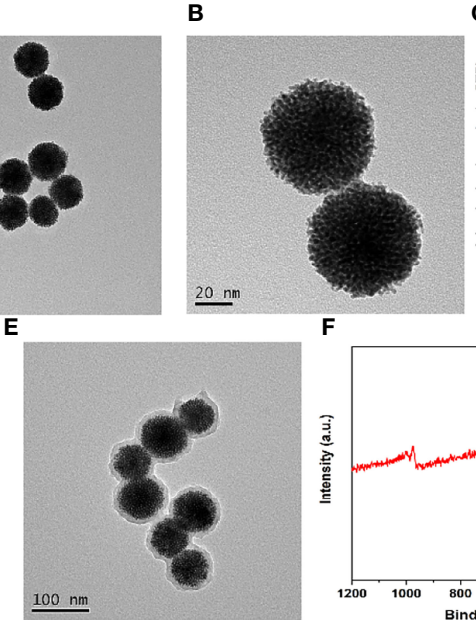

H
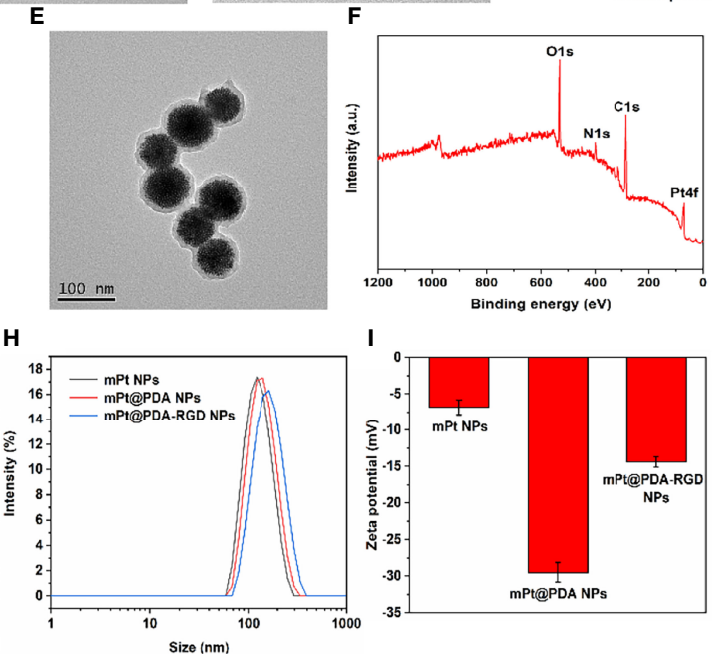

C

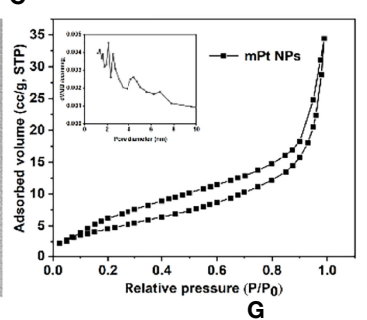

D

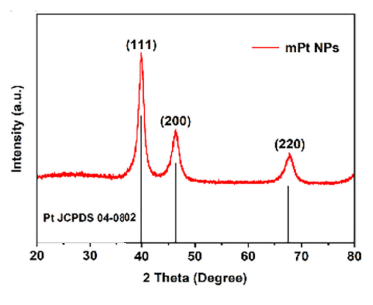

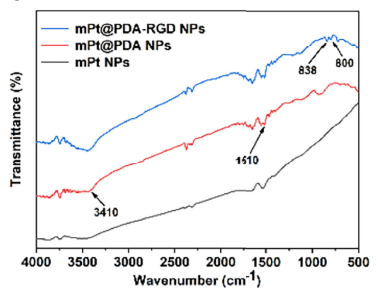

J

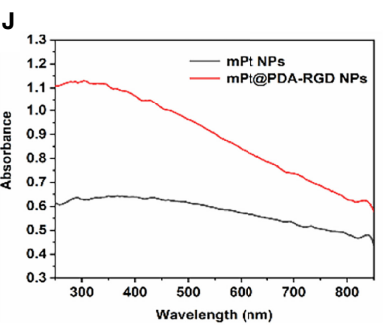

FIGURE 1 | Transmission Electron Microscope (TEM) images of mPt NPs (A, B). Pore size distribution (C) and X-ray diffraction (XRD) patterns (D) of mPt NPs. (E) TEM image of mPt@PDA NPs. X-ray photoelectron spectroscopy (XPS) spectra (F) of mPt@PDA NPs. (G) Fourier transform infrared spectroscopy (FTIR) of mPt@PDA NPs and mPt@PDA-RGD NPs. Size distribution (H) and Zeta potential (I) of mPt NPs, mPt@PDA NPs, and mPt@PDA-RGD NPs. (J) Uv-visible absorption spectrum (UV-Vis) spectral of mPt NPs and mPt@PDA-RGD NPs. 
mesoporous shape was further confirmed by $\mathrm{N}_{2}$ absorptiondesorption. As shown in Figure 1C, $\mathrm{mPt}$ NPs displayed wide pore diameter distribution (about 2-8 nm) and high surface area (46.76 $\mathrm{m}^{2} / \mathrm{g}$ ). The XRD pattern of the mPt NPs (Figure 1D) was highly consistent with that of bulk Pt (JCPDS 04-0802). After PDA coating, the prepared mPt@PDA NPs showed a core/shell morphology (Figure 1E), and XPS spectra (Figure 1F) of mPt@PDA NPs further confirmed the presence of Pt elements. As shown in Figure 1G, the wide absorption bands at $3,410 \mathrm{~cm}^{-1}$ could be attributed to the stretching vibration of phenolic $\mathrm{O}-\mathrm{H}$ and $\mathrm{N}-\mathrm{H}$. The bands at $1,510 \mathrm{~cm}^{-1}$ corresponded to shearing vibration of $\mathrm{N}-\mathrm{H}$. The above absorption bands on mPt@PDA NPs indicated the successful polymerization of PDA. Meanwhile, the new peaks of mPt@PDA-RGD NPs at 800 and $838 \mathrm{~cm}^{-1}$, which belonged to the peaks of phenyl group of RGD, confirmed the successful grafting of RGD. The diameter of NPs in aqueous solution was determined by Dynamic Light Scattering (DLS) method. The results (Figure 1H) showed that the average hydrodynamic diameter of mPt NPs, mPt@ PDA NPs, and mPt@PDA-RGD NPs was about 124.5, 133.9, and $151.6 \mathrm{~nm}$, respectively. Zeta potential (Figure 1I) shows that PDAmodified mPt NPs significantly reduced the potential, which was due to the hydroxyl group on the surface of PDA. The potential of mPt@ PDA-RGD NPs was increased compared with that of mPt@PDA NPs, which was attributed to the positively charged amino group on RGD. UV-vis absorption spectra (Figure 1J) showed that mPt@ PDA-RGD NPs had extensive absorption at the NIR region, confirming the good potential of mPt@PDA-RGD NPs as effective photosensitizers.

\section{Photothermal Effect Evaluation of mPt@ PDA-RGD Nanoparticles}

Various concentrations of mPt@PDA-RGD NP aqueous solution were irradiated with $808 \mathrm{~nm}\left(1.5 \mathrm{~W} / \mathrm{cm}^{2}, 5 \mathrm{~min}\right)$; meanwhile, the temperature of samples was monitored. Figure $2 \mathbf{A}$ shows that the temperature increased with the increase of nanomaterial concentration. In contrast, the temperature of $\mathrm{H}_{2} \mathrm{O}$ was almost constant under the same irradiation conditions. Under a fixed concentration of mPt@PDA-RGD NPs $(100 \mu \mathrm{g} / \mathrm{ml})$, the change of temperature was positively correlated with the optical density
(Figure 2B). In general, the increase of NP temperature showed the dependence of solution concentration and optical density. In addition, we evaluated the photothermal stability of the NPs by performing four cycles of "laser-on/off" experiments, during which mPt@PDA-RGD NPs were irradiated for $5 \mathrm{~min}$ and cooled naturally. Figure 2C shows after 4 cycles that the temperature did not decrease obviously, indicating that NPs had good photothermal stability. Thus, mPt@PDA-RGD NPs show excellent photothermal properties under 808-nm laser irradiation.

\section{Cellular Uptake Experiment}

SKOV-3 cells were coincubated with mPt@PDA or mPt@PDARGD NPs for different times to detect the concentration of $\mathrm{Pt}$ internalization by ICP-OES. As shown in Figure 3D, the absorption of $\mathrm{Pt}$ elements was of positive relevance with the incubation time of NPs and SKOV-3 cells. In addition, more Pt elements were detected in cells treated with mPt@PDA-RGD NPs than in those treated with mPt@PDA NPs. These proved that mPt@PDA-RGD NPs have effective targeting ability to SKOV -3 cells.

\section{In Vitro Photothermal Therapy Effects of mPt@PDA-RGD Nanoparticles}

To evaluate the cytotoxicity of mPt NPs and mPt@PDA-RGD NPs, CCK- 8 assay was used to evaluate the cell viability, and the results displayed that $\mathrm{mPt}$ NPs showed poor biocompatibility at high concentrations (Figure 3A); however, after modification by PDA coating, mPt@PDA-RGD NPs had no significant toxicity on SKOV-3 cells under the concentration of $100 \mu \mathrm{g} / \mathrm{ml}$ (Figure 3B). Even when reaching $100 \mu \mathrm{g} / \mathrm{ml}$, the viability of SKOV-3 cells was still above $80 \%$, indicating that mPt NPs had obvious toxicity to cells, but the modification of PDA greatly reduced the toxicity of $\mathrm{mPt} N \mathrm{NP}$ and improved the cytocompatibility of the NPs. We also tested the cytotoxicity of mPt@PDA-RGD NPs under PTT. Figure 3C shows no obvious cytotoxicity in the control group, laser group, and mPt@PDARGD NP group. However, the cell survival rate of the mPt@ PDA-RGD NPs+laser group was reduced to about 29\%, which
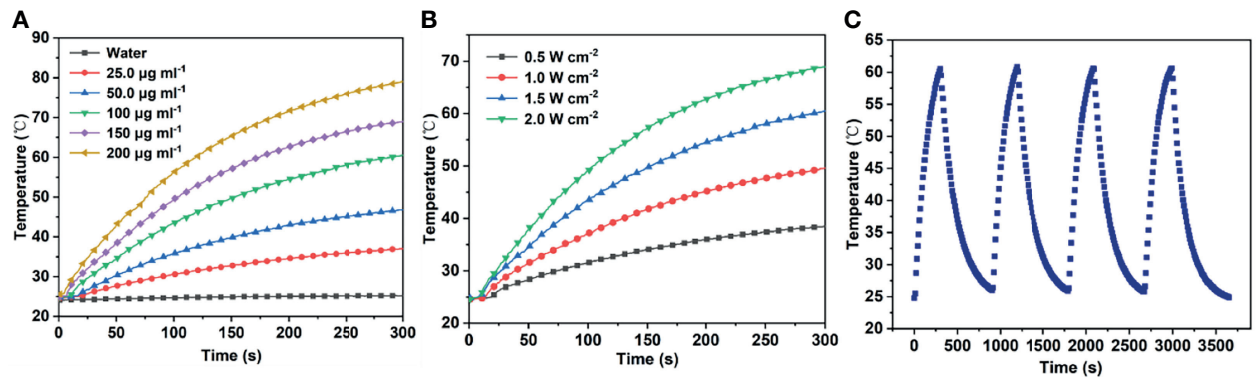

FIGURE 2 | (A) Temperature responses of mPt@PDA-RGD NP solutions with various Pt concentrations under the 808-nm laser. (B) Temperature responses of mPt@PDA-RGD NP solutions (100 $\mu \mathrm{g} / \mathrm{ml})$ under the 808-nm laser with different power densities for $300 \mathrm{~s}$. (C) The heating curve of the mPt@PDA-RGD NP dispersion in deionized water at 4 laser on/off cycles $\left(1.5 \mathrm{~W} / \mathrm{cm}^{2}\right)$ under $808-\mathrm{nm}$ laser irradiation. 

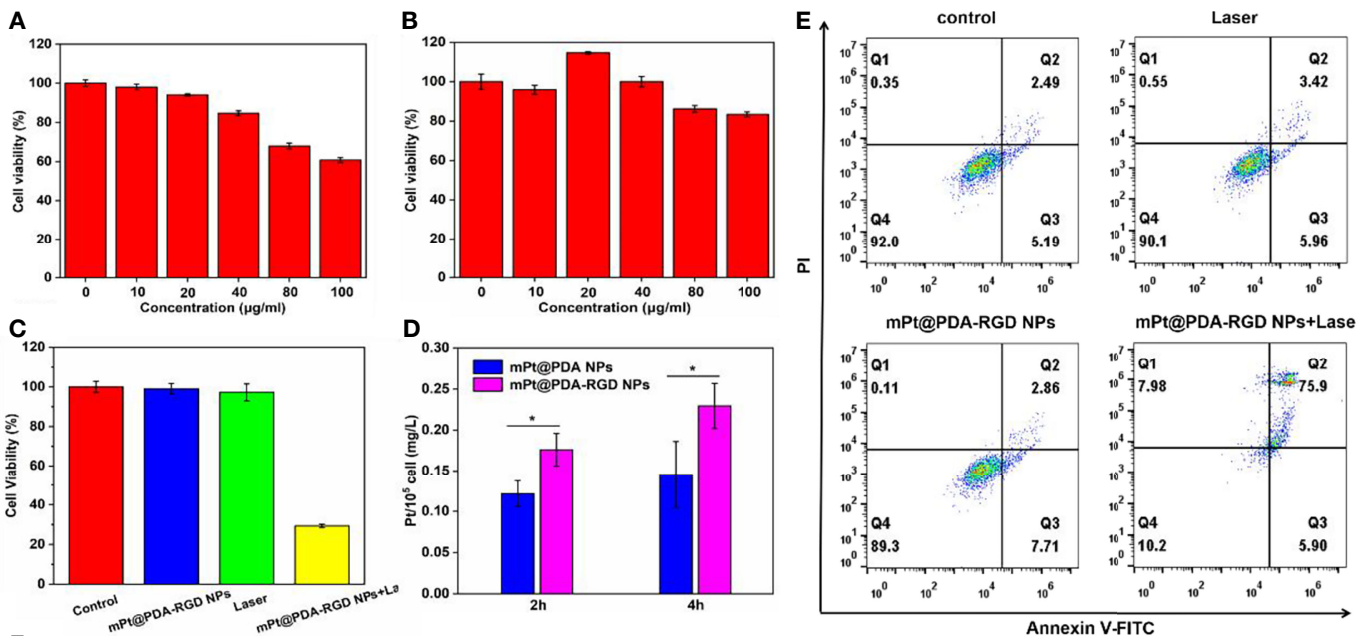

mPt@PDA-RGD NPS
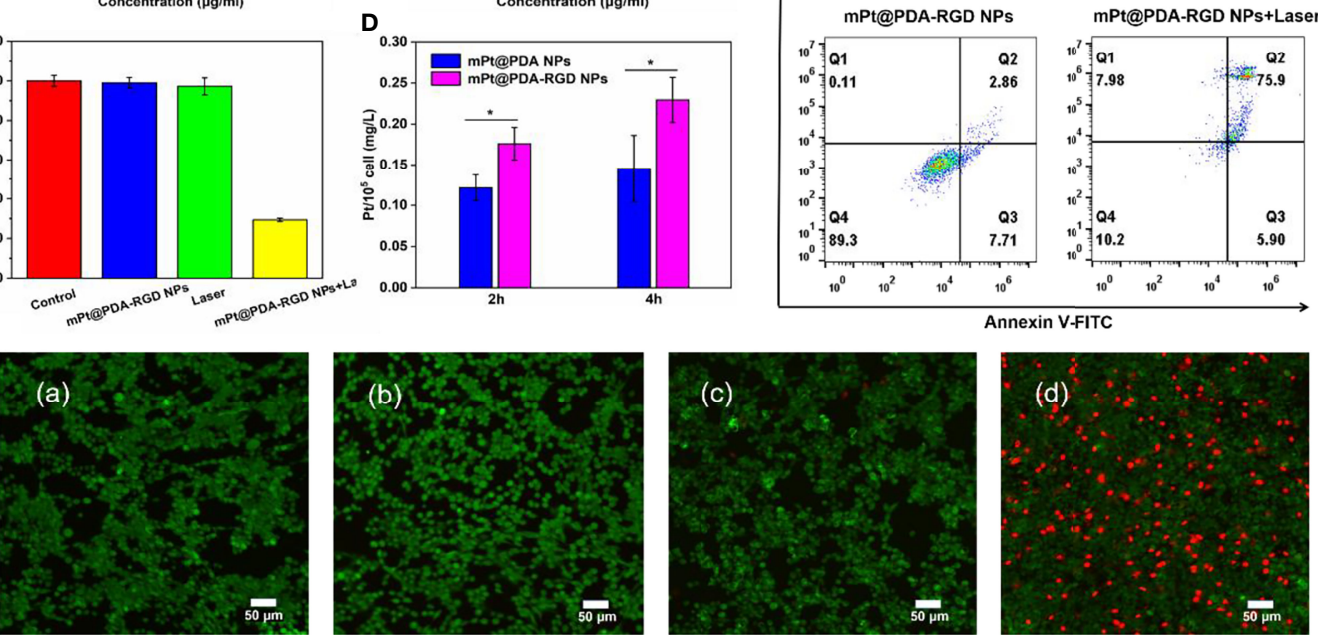

FIGURE 3 | Cytotoxicity of (A) mPt NPs and (B) mPt@PDA-RGD NPs. (C) Relative viability of SKOV-3 cells treated with mPt@PDA-RGD NPs and laser. (D) Pt contents in SKOV-3 cells with different treatments. All values are presented as means \pm standard errors of the mean (SEM); ${ }^{*} \mathrm{P}<0.05$. (E) Flow cytometry analysis of SKOV-3 cells. (F) Calcein AM/PI staining of SKOV-3 cells with various treatments: (a) control, (b) laser (808 nm), (c) mPt@PDA-RGD NPs, (d) mPt@PDA-RGD NPs+laser.

reflected the excellent photothermal performance of mPt@PDARGD NPs.

Flow cytometry was further performed to quantify cell viability. mPt@PDA-RGD NPs $(50 \mu \mathrm{g} / \mathrm{ml})$ were cultured with SKOV-3 cells for $24 \mathrm{~h}$. Next, cells were washed two times with PBS, new medium was refreshed, and cells were exposed to 808$\mathrm{nm}$ laser irradiation; Annexin V and PI double staining was used to detect cytotoxicity. Figure 3E shows that the cell viability of the control, laser, and mPt@PDA-RGD NP groups was maintained at about $90 \%$. However, the cell viability dropped to $10.2 \%$ in the mPt@PDA-RGD+laser group. The results show that mPt@PDA-RGD NPs can induce apoptosis significantly under NIR laser and is a highly efficient photothermal agent.

Moreover, Calcein AM/PI staining was used to further verify the PTT efficiency of mPt@PDA-RGD NPs. SKOV-3 cells in the control group, laser group, and mPt@PDA NP group showed bright fluorescent green, indicating the survival status of cells. However, the mPt@PDA-RGD NPs with laser group observed the most red fluorescence (Figure 3F), suggesting that PTT was effective in killing cancer cells.

The effects of mPt@PDA-RGD NPs and PTT on actin cytoskeleton in SKOV-3 cells were further analyzed by CLSM. As shown in Figure 4A, the actin filaments in the cytoplasm of the control group showed highly ordered cytoskeleton arrangement with thick bundle distribution. The actin filaments in cytoplasm showed no obvious change after laser treatment, indicating that only laser treatment had almost no influence on the cytoskeleton. Interestingly, SKOV-3 cells with mPt@PDA-RGD NP treatment showed partial curling of the actin filaments at the cell edge, and the curling degree of actin filaments displayed obvious mPt@PDA-RGD NPs dosedependent manner. When SKOV-3 cells were synchronously treated with mPt@PDA-RGD NPs and laser, the fluorescence intensity of actin filaments was obviously weakened, and the structure of F-actin contracted and curled seriously, losing the ordered arrangement state. Western blot analysis was further performed to verify the change of actin expression. As shown in Figure 4B, the expression of actin in SKOV-3 cells was significantly downregulated with the increase of mPt@PDARGD NP dose under the same laser intensity. These results indicated that the photothermal effect produced by mPt@PDARGD NPs+laser treatment can effectively disturb the cytoskeleton.

Subsequently, cell scratch experiments were used to investigate the antimigration performance of mPt@PDA-RGD NPs under laser. As shown in Figures 4C, D, there was no significant difference in the cell migration between the NIR group and the control group at $24 \mathrm{~h}$, indicating that only laser irradiation had no significant effect on inhibiting migration. While the mPt@PDA-RGD NP group displayed a dosedependent SKOV-3 cell migration inhibition, which was 
A
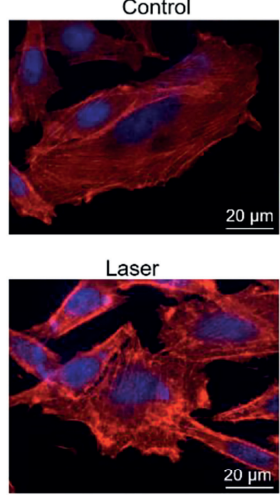

D
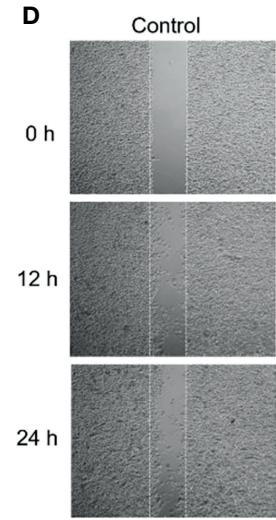

$50 \mu \mathrm{g} / \mathrm{mL}$

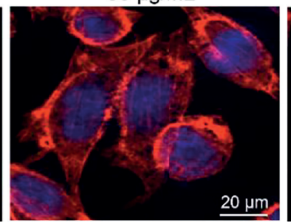

$50 \mu \mathrm{g} / \mathrm{mL}+$ Laser

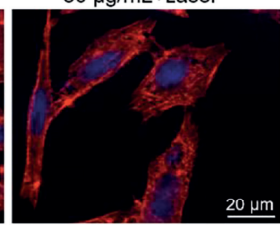

$100 \mu \mathrm{g} / \mathrm{mL}$

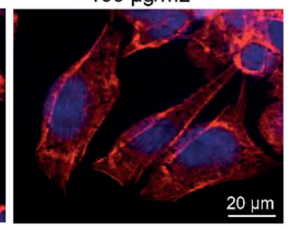

$100 \mu \mathrm{g} / \mathrm{mL}+$ Laser

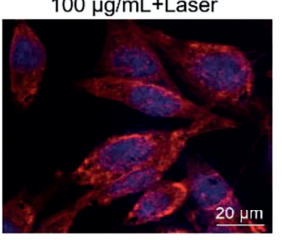

$\underline{20 \mu m}$

$50 \mu \mathrm{g} / \mathrm{mL}+$ Laser
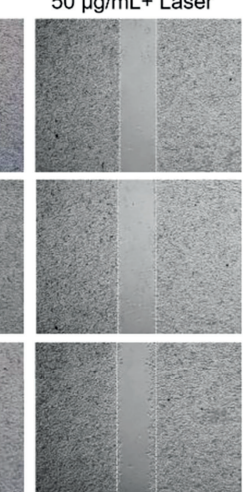

B

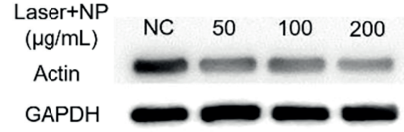

GAPDH

C
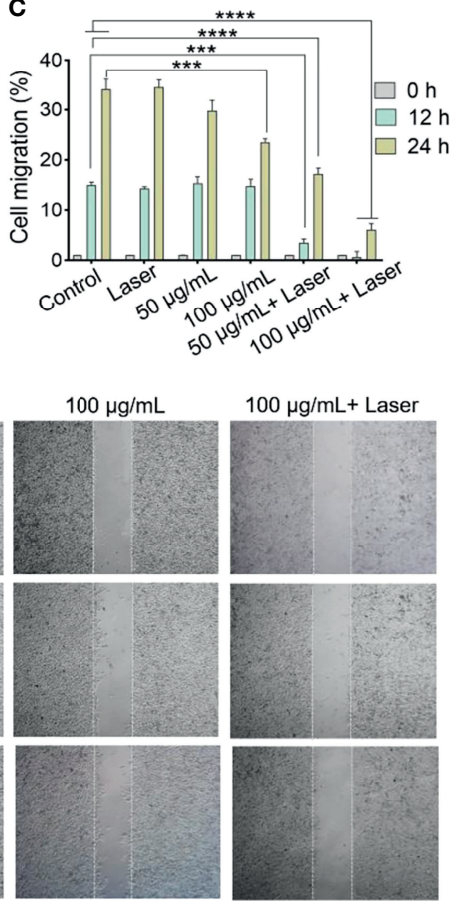

FIGURE 4 | (A) Immunofluorescence images and (B) Western blot analysis of SKOV-3 actin staining after various treatments. (C) Corresponding migration rate. All values are presented as means \pm standard errors of the mean (SEM); ${ }^{\star \star \star *} p<0.0001,{ }^{\star \star \star} p<0.001$. (D) The migration performance of SKOV-3 cells with various treatments.

attributed to the disturbance of cytoskeleton by mPt@PDA-RGD NPs. With laser irradiation treatment, the cell migration of the mPt@PDA-RGD NP group was significantly reduced both at 12 and $24 \mathrm{~h}$, indicating that the photothermal effect of mPt@PDARGD NPs produced a significant inhibitory effect on the cell migration of SKOV-3 cells.

PTT has been widely used in basic research and preclinical field. There are a few studies using porous Pt nanoparticles for PTT, and some limitations exist. For example, Zhu et al. (35) synthesized porous Pt nanoparticles coated with poly (diallyl dimethyl ammonium chloride) for PTT. More than $70 \%$ of the cells were phagocytosed by photothermal irradiation of $808-\mathrm{nm}$ laser at $8.4 \mathrm{~W} / \mathrm{cm}^{2}$ for $3 \mathrm{~min}$. The photothermal effect is obvious, but the high laser power greatly limits the clinical application of the material. The mPt@PDA-RGD NPs synthesized by us have obvious photothermal effect under $808-\mathrm{nm}$ laser at $1.5 \mathrm{~W} / \mathrm{cm}^{2}$, which can effectively kill most tumor cells at a relatively safe power. Moreover, we found that the photothermal effect can destroy the actin cytoskeleton and inhibit tumor migration, which is rarely reported. This study provides a new direction for PTT of tumors.

\section{CONCLUSIONS}

In conclusion, we developed a multifunctional PTT nanoplatform based on mPt@PDA-RGD NPs. As a photosensitizer, mPt was the core of NPs, and its surface was coated with PDA, which improves biocompatibility and facilitates functional modification of NPs.

Next, mPt@PDA NPs with RGD coupling can target highly expressed integrin $\alpha_{\mathrm{v}} \beta_{3}$ on SKOV-3 cells through receptormediated targeting. Cell experiments showed that mPt@PDARGD NPs can not only effectively induce tumor ablation but also greatly inhibit the migration ability of surviving cancer cells, which was attributed to the disturbance of actin cytoskeleton under the photothermal effect. Thus, mPt@PDA-RGD NPs showed great potential as an effective PTT nanomedicine for tumors targeted by photothermal ablation and migration inhibition.

\section{DATA AVAILABILITY STATEMENT}

The original contributions presented in the study are included in the article/supplementary material. Further inquiries can be directed to the corresponding authors. 


\section{AUTHOR CONTRIBUTIONS}

JD: resources, supervision, project administration, and funding acquisition. XS: experiment design, supervision, validation, and funding acquisition. HW: conceptualization, methodology, resources, and supervision. XZ: formal analysis, data curation, visualization, and writing. GM: formal analysis, data curation, visualization, and writing. PZ: visualization and investigation. YC: visualization and investigation. All authors contributed to the article and approved the submitted version.

\section{REFERENCES}

1. Liu Y, Bhattarai P, Dai Z, Chen X. Photothermal Therapy and Photoacoustic Imaging via Nanotheranostics in Fighting Cancer. Chem Soc Rev (2019) 48 (7):2053-108. doi: 10.1039/c8cs00618k

2. Wang XW, Shi QQ, Zha ZB, Zhu DD, Zheng LR, Shi LX, et al. Copper SingleAtom Catalysts With Photothermal Performance and Enhanced Nanozyme Activity for Bacteria-Infected Wound Therapy. Bioact Mater (2021) 6:4389401. doi: 10.1016/j.bioactmat.2021.04.024

3. Wang XW, Cheng L. Multifunctional Prussian Blue-Based Nanomaterials: Preparation, Modification, and Theranostic Applications. Coordin Chem Rev (2020) 419:213393. doi: 10.1016/j.ccr.2020.213393

4. Jiang S, Liu Z, Tian YH, Zhuang M, Piao SQ, Gao Y, et al. A Comprehensive Evaluation of ZrC Nanoparticle in Combined Photothermal and Radiation Therapy for Treatment of Triple-Negative Breast Cancer. Front Oncol (2021) 11:801352:801352. doi: 10.3389/fonc.2021.801352

5. Vankayala R, Hwang KC. Near-Infrared-Light-Activatable NanomaterialMediated Phototheranostic Nanomedicines: An Emerging Paradigm for Cancer Treatment. Adv Mater (2018) 30(23):e1706320:e1706320. doi: 10.1002/adma.201706320

6. Xu L, Mou F, Gong H, Luo M, Guan J. Light-Driven Micro/Nanomotors: From Fundamentals to Applications. Chem Soc Rev (2017) 46(22):6905-26. doi: $10.1039 / \mathrm{c} 7 \mathrm{cs} 00516 \mathrm{~d}$

7. Gai S, Yang G, Yang P, He F, Lin J, Jin D, et al. Recent Advances in Functional Nanomaterials for Light-Triggered Cancer Therapy. Nano Today (2018) 19:146-87. doi: 10.1016/j.nantod.2018.02.010

8. Wang XW, Zhong XY, Cheng L. Titanium-Based Nanomaterials for Cancer Theranostics. Coordin Chem Rev (2020) 430:213662. doi: 10.1016/ j.ccr.2020.213662

9. Ni NY, Zhang XY, Ma YL, Yuan J, Wang DQ, Ma GQ, et al. Biodegradable Two-Dimensional Nanomaterials for Cancer Theranostics. Coordin Chem Rev (2022) 458:214415. doi: 10.1016/j.ccr.2022.214415

10. Wang XW, Wang XY, Yue QF, Xu HZ, Zhong XY, Sun LN, et al. Liquid Exfoliation of TiN Nanodots as Novel Sonosensitizers for PhotothermalEnhanced Sonodynamic Therapy Against Cancer. Nano Today (2021) 39:101170. doi: 10.1016/j.nantod.2021.101170

11. Sun X, Ni NY, Ma YL, Wang Y, Leong DT. Retooling Cancer Nanotherapeutics' Entry Into Tumors to Alleviate Tumoral Hypoxia. Small (2020) 16(41):2003000. doi: 10.1002/smll.202003000

12. Ni NY, Su YQ, Wei YC, Ma YL, Zhao LZ, Sun X. Tuning Nanosiliceous Framework for Enhanced Cancer Theranostic Applications. Adv Ther (2021) 4(4):2000218. doi: 10.1002/adtp.202000218

13. Mu M, Wang YL, Zhao SS, Li XL, Fan RR, Mei L, et al. Engineering a Ph/ Glutathione-Responsive Tea Polyphenol Nanodevice as an Apoptosis/ Ferroptosis-Inducing Agent. ACS Appl Bio Mater (2020) 3(7):4128-38. doi: 10.1021/acsabm.0c00225

14. Xie RX, Wu ZJ, Zeng FX, Cai HW, Wang D, Gu L, et al. Retro-Enantio Isomer of Angiopep-2 Assists Nanoprobes Across the Blood-Brain Barrier for Targeted Magnetic Resonance/Fluorescence Imaging of Glioblastoma. Signal Transduct Tar (2021) 6:309. doi: 10.1038/s41392-021-00724-y

\section{FUNDING}

This work was supported by the National Natural Science Foundation of China (22104073); Natural Science Foundation of Shandong (ZR2020MB073, ZR2021QB119); Shandong Health System Outstanding Young Talent Project, Overseas Science and Technology Talents Project of Shandong Province, Academic Promotion Program of Shandong First Medical University (2019QL008); and the Youth Outstanding Reserve Talents Program of Shandong First Medical University and Shandong Academy of Medical Sciences.

15. Shanmugam V, Selvakumar S, Yeh CS. Near-Infrared Light-Responsive Nanomaterials in Cancer Therapeutics. Chem Soc Rev (2014) 43(17):625487. doi: $10.1039 / \mathrm{c} 4 \mathrm{cs} 00011 \mathrm{k}$

16. Wang S, Xin J, Zhang L, Zhou Y, Yao C, Wang B, et al. CantharidinEncapsulated Thermal-Sensitive Liposomes Coated With Gold Nanoparticles for Enhanced Photothermal Therapy on A431 Cells. Int J Nanomed (2018) 13:2143-60. doi: 10.2147/IJN.S156240

17. Riley RS, Day ES. Gold Nanoparticle-Mediated Photothermal Therapy: Applications and Opportunities for Multimodal Cancer Treatment. Wiley Interdiscip Rev Nanomed Nanobiotechnol (2017) 9(4):e1449. doi: 10.1002/ wnan.1449

18. Huang X, Yin YL, Wu M, Zan W, Yang Q. LyP-1 Peptide-Functionalized Gold Nanoprisms for SERRS Imaging and Tumor Growth Suppressing by PTT Induced-Hyperthermia. Chin Chem Lett (2019) 30:1335-40. doi: 10.1016/ j.cclet.2019.02.019

19. Dickerson EB, Dreaden EC, Huang X, El-Sayed IH, Chu H, Pushpanketh S, et al. Gold Nanorod Assisted Near-Infrared Plasmonic Photothermal Therapy (PPTT) of Squamous Cell Carcinoma in Mice. Cancer Lett (2008) 269(1):5766. doi: 10.1016/j.canlet.2008.04.026

20. Christopher Loo AL, Halas N, West J, Drezek R. Immunotargeted Nanoshells for Integrated Cancer Imaging and Therapy. Nano Lett (2005) 5(4):709-11. doi: $10.1021 / \mathrm{nl} 050127 \mathrm{~s}$

21. Skrabalak SE, Chen J, Au L, Lu X, Li X, Xia Y. Gold Nanocages for Biomedical Applications. Adv Mater (2007) 19(20):3177-84. doi: 10.1002/adma.200701972

22. Zhang JZ. Biomedical Applications of Shape-Controlled Plasmonic Nanostructures: A Case Study of Hollow Gold Nanospheres for Photothermal Ablation Therapy of Cancer. J Phys Chem Lett (2010) 1 (4):686-95. doi: 10.1021/jz900366c

23. Manikandan M, Hasan N, Wu HF. Platinum Nanoparticles for The Photothermal Treatment of Neuro 2a Cancer Cells. Biomaterials (2013) 34 (23):5833-42. doi: 10.1016/j.biomaterials.2013.03.077

24. Mrowczynski R. Polydopamine-Based Multifunctional (Nano)Materials for Cancer Therapy. ACS Appl Mater Inter (2018) 10(9):7541-61. doi: 10.1021/ acsami.7b08392

25. Zhou Y, Lin BX, Li K, Zhao YF, Sun Z, He CC, et al. Preparation of NearInfrared/Photoacoustic Dual-Mode Imaging and Photothermal/Chemo Synergistic Theranostic Nanoparticles and Their Imaging and Treating of Hepatic Carcinoma. Front Oncol (2021) 11:750807:750807. doi: 10.3389/ fonc. 2021.750807

26. Li K, Li RY, Zhou BN, Chen J, Lan K, Zhan WH, et al. Cascade Release Nanocarriers for the Triple-Negative Breast Cancer Near-Infrared Imaging and Photothermal-Chemo Synergistic Therapy. Front Oncol (2021) 11:747608:747608. doi: 10.3389/fonc.2021.747608

27. Cheng W, Zeng X, Chen H, Li Z, Zeng W, Mei L, et al. Versatile Polydopamine Platforms: Synthesis and Promising Applications for Surface Modification and Advanced Nanomedicine. ACS Nano (2019) 13(8):8537-65. doi: 10.1021/ acsnano.9b04436

28. Li Z, Hu Y, Howard KA, Jiang T, Fan X, Miao Z, et al. Multifunctional Bismuth Selenide Nanocomposites for Antitumor Thermo-Chemotherapy and Imaging. ACS Nano (2016) 10(1):984-97. doi: 10.1021/acsnano.5b06259 
29. Liu XS, Cao JM, Li H, Li JY, Jin Q, Ren KF, et al. Mussel-Inspired Polydopamine: A Biocompatible and Ultrastable Coating for Nanoparticles In Vivo. ACS Nano (2013) 7(10):9384-95. doi: 10.1021/nn404117j

30. Park J, Brust TF, Lee HJ, Lee SC, Watts VJ, Yeo Y. Polydopamine-Based Simple and Versatile Surface Modification of Polymeric Nano Drug Carriers. ACS Nano (2014) 8(4):3347-56. doi: 10.1021/nn405809c

31. Polarek W, G JR, D T. Characterization of A Hyaluronic Acid-Arg-Gly-Asp Peptide Cell Attachment Matrix. Biomaterials (1996) 17(11):1101-8. doi: 10.1016/0142-9612(96)85911-4

32. Liao YT, Liu CH, Chin Y, Chen SY, Liu SH, Hsu YC, et al. Biocompatible and Multifunctional Gold Nanorods for Effective Photothermal Therapy of Oral Squamous Cell Carcinoma. J Mater Chem B (2019) 7(28):4451-60. doi: $10.1039 /$ c9tb00574a

33. Guo W, Giancotti FG. Integrin Signalling During Tumour Progression. Nat Rev Mol Cell Biol (2004) 5(10):816-26. doi: 10.1038/nrm1490

34. Li Y, Yun KH, Lee H, Goh SH, Suh YG, Choi Y. Porous Platinum Nanoparticles as A High-Z and Oxygen Generating Nanozyme for Enhanced Radiotherapy In Vivo. Biomaterials (2019) 197:12-9. doi: 10.1016/j.biomaterials.2019.01.004

35. Zhu XM, Wan HY, Jia HL, Liu L, Wang JF. Porous Pt Nanoparticles With High Near-Infrared Photothermal Conversion Efficiencies for Photothermal
Therapy. Adv Healthc Mater (2016) 5(24):3165-72. doi: 10.1002/ adhm. 201601058

Conflict of Interest: The authors declare that the research was conducted in the absence of any commercial or financial relationships that could be construed as a potential conflict of interest.

Publisher's Note: All claims expressed in this article are solely those of the authors and do not necessarily represent those of their affiliated organizations, or those of the publisher, the editors and the reviewers. Any product that may be evaluated in this article, or claim that may be made by its manufacturer, is not guaranteed or endorsed by the publisher.

Copyright (c) $2022 \mathrm{Zou}, \mathrm{Ma}, \mathrm{Zhu}$, Cao, Sun, Wang and Dong. This is an open-access article distributed under the terms of the Creative Commons Attribution License (CC BY). The use, distribution or reproduction in other forums is permitted, provided the original author(s) and the copyright owner(s) are credited and that the original publication in this journal is cited, in accordance with accepted academic practice. No use, distribution or reproduction is permitted which does not comply with these terms. 